\title{
The Time-Varying Correlation between Crude oil Future and USA Bond Markets During 2005-2020: Evidence from a DCC-GARCH Model
}

Konstantinos Tsiaras

University of Ioannina, PhD, University Campus, 54110, Ioannina, Greece

\author{
DOI: $10.36348 /$ sjef.2020.v04i12.003 \\ | Received: 26.11 .2020 | Accepted: 08.12.2020 | Published: 13.12.2020
}

*Corresponding author: Konstantinos Tsiaras

\section{Abstract}

In this paper, we examine potential time-varying correlations between crude oil future and USA bond markets. We employ a dynamic conditional correlation (DCC) multivariate GARCH model in order to quantify potential contagion effects between the markets for the period 2005-2020. We divide the period in two sub-period to make the empirical analysis easier. Empirical results reveal increased conditional correlation in the first sub-period (2005-2012) and no contagion in the second sub-period (2012-2020). Results are of interest to investors, who invest long-term into the under investigation financial markets, as well as, to policymakers, who provide regulations for the under investigation derivate market.

Keywords: DCC-GARCH, Crude oil future market, USA bond market, financial contagion, dynamic conditional correlations.

Jel Classification: C58, C61, G10.

Copyright $\odot \mathbf{2 0 2 0}$ The Author(s): This is an open-access article distributed under the terms of the Creative Commons Attribution 4.0 International License (CC BY-NC 4.0) which permits unrestricted use, distribution, and reproduction in any medium for non-commercial use provided the original author and source are credited.

\section{INTRODUCTION}

In this paper we empirically investigate the spillovers and contagion between crude oil future market and six USA bond markets from $3^{\text {rd }}$ January 2005 to $31^{\text {st }}$ August 2012, including the USA subprime crisis, and from $3^{\text {rd }}$ September 2012 to $27^{\text {th }}$ August 2020, including the European Sovereign Debt Crisis. To quantify volatility spillover effects, we use a multivariate DCC-GARCH process [1]. According to Forbes and Rigobon [2] contagion is defined as a significant increase in cross-market linkages after a shock. We use the produced dynamic conditional correlations to measure contagion. Our empirical findings support the conclusion that investors can benefit from diversifying into the under investigation financial markets. Furthermore, the policy implications of the empirical results are also crucial. Policymakers can expect the markets to recover without additional measures are taken.

In recent years it has been noted that the effects of crude oil market influence major economic activity [3-7]. It has been identified the effects of crude oil market returns on stock returns [8-14]. A few studies have discussed the effects of crude oil market on bond markets $[15,16]$. There are several studies investigating the linkages between crude oil future market and financial markets [17-23]. To the best of our knowledge this is the first such investigation of spillovers and contagion between crude oil future and USA bond markets.

Our paper adds to the related literature in several ways. First, we investigate the dynamic links between crude oil future and USA bond markets based on a time-varying framework, which had not been researched before in the literature. We examine whether the crude oil future market returns is exposed to volatility in USA bond markets. Based on dynamic links, we present the existence of potential contagion effects. The sample period allowed us to investigate the potential effects of major economic events on the dynamic links between the markets.

The remainder of this paper is organized as follows: Section two presents the methodology. Section three analyzes the empirical results. Section four gives concluding comments.

\section{METHODOLODY \\ Model Description}

First we define the daily logarithmic returns:

$$
\mathrm{y}_{\mathrm{t}}=\mu+\varepsilon_{\mathrm{t}} \text {, with } \mathrm{t}=1, \ldots, \mathrm{T}
$$

Where $\mu$ is constant and $\varepsilon_{\mathrm{t}}$ is standardized residuals defined as follows: 
$\varepsilon_{\mathrm{t}}=\sqrt{\mathrm{h}_{\mathrm{t}}} \mathrm{u}_{\mathrm{t}}$, where $\varepsilon_{\mathrm{t}} \sim \mathrm{N}\left(0, \mathrm{H}_{\mathrm{t}}\right)$ and $\mathrm{u}_{\mathrm{t}}$ are i.i.d. (2)

Where $u_{t}$ is standardized errors and $h_{t}$ is conditional variance depending on $h_{t}$ and $\varepsilon_{t}$ for each market lagged one period, generated by the univariate GARCH(1,1) model [24]:

$$
h_{t}=\omega+a \varepsilon_{t-1}^{2}+b h_{t-1}
$$

Where $\omega$ is constant, $a$ and $b$ are ARCH and GARCH effects.

Next, we employ the Engle [1] representation of the bivariate GARCH model in order to estimate the bivariate conditional variance matrix $\left(\mathrm{H}_{t}\right.$ is $\mathrm{N} \times \mathrm{N}$ matrix, with $\mathrm{N}$ the number of markets, $\mathrm{i}=1, \ldots, \mathrm{N})$ as follows:

$$
\mathrm{H}_{\mathrm{t}}=\mathrm{D}_{\mathrm{t}} \mathrm{R}_{\mathrm{t}} \mathrm{D}_{\mathrm{t}}
$$

$D_{t}$ is the conditional variance matrix given by:

$$
\mathrm{D}_{\mathrm{t}}=\operatorname{diag}\left(\mathrm{h}_{11 \mathrm{t}}^{\frac{1}{2}} \ldots \mathrm{h}_{\mathrm{NN \textrm {t }}}^{\frac{1}{2}}\right)
$$

$\mathrm{R}_{\mathrm{t}}$ is the condition correlation matrix of $\mathrm{N} \times \mathrm{N}$ dimension, and is defined as follows:

$$
\begin{gathered}
\mathrm{R}_{\mathrm{t}}=\left(\rho_{\text {iit }}\right)= \\
\operatorname{diag}\left(\mathrm{q}_{11, \mathrm{t}}^{-\frac{1}{2}} \ldots \mathrm{q}_{\mathrm{NN}, \mathrm{t}}^{-\frac{1}{2}}\right) \mathrm{Q}_{\mathrm{t}} \operatorname{diag}\left(\mathrm{q}_{11, \mathrm{t}}^{-\frac{1}{2}} \ldots \mathrm{q}_{\mathrm{NN}, \mathrm{t}}^{-\frac{1}{2}}\right)
\end{gathered}
$$

Where the $\mathrm{N} \times \mathrm{N}$ symmetric positive definite matrix $\mathrm{Q}_{\mathrm{t}}=\left(\mathrm{q}_{\mathrm{ii}, \mathrm{t}}\right)$ is given by:

$$
\mathrm{Q}_{\mathrm{t}}=(1-\alpha-\beta) \overline{\mathrm{Q}}+\alpha \mathrm{u}_{\mathrm{t}-1} \mathrm{u}_{\mathrm{t}-1}^{\prime}+\beta \mathrm{Q}_{\mathrm{t}-1}, \ldots
$$

$\overline{\mathrm{Q}}$ is the $\mathrm{N} \times \mathrm{N}$ unconditional variance matrix of $\mathrm{u}_{\mathrm{t}}$, and $\alpha$ and $\beta$ are nonnegative scalar parameters, satisfying $\alpha$ $+\beta<1$.

\section{Data Description}

In this paper, we use daily data for NYMLIGHT CRUDE OIL CONT.INDEX - SETT. PRICE, US BENCHMARK 2 YEAR DS GOVT. INDEX CLEAN PRICE INDEX, US BENCHMARK 3 YEAR DS GOVT. INDEX - CLEAN PRICE INDEX, US BENCHMARK 5 YEAR DS GOVT. INDEX - CLEAN PRICE INDEX, US BENCHMARK 7 YEAR DS GOVT. INDEX - CLEAN PRICE INDEX, US BENCHMARK 10 YEAR DS GOVT. INDEX CLEAN PRICE INDEX and US BENCHMARK 30 YEAR DS GOVT. INDEX - CLEAN PRICE INDEX. Based on the Global Financial Crisis of 2008, we divide the sample period into two sub-periods: the first period from 03/01/2005 until 31/08/2012 (2000 obs.) and the second period from 03/09/2012 until 27/08/2020 (2084 obs). We extracted the data from Datastream ${ }^{\circledR}$ Database. We generate market logarithmic returns using the equation: $y r_{t}=\log \left(p_{t}\right)-\log \left(p_{t-1}\right)$, where $p_{t}$ is the price of market on day $t$.

Figure 1 and 2 show the dynamics of market logarithmic returns during the under investigation periods. We observe the presence of volatility clustering and $\mathrm{ARCH}$ effects in all series, rationalizing the adoption of the GARCH models.

\section{RESULTS AND DISCUSSION}

In this section, we present the empirical results for the two under investigation periods: 3 January, 2005 - 31 August, 2012 and 3 September, 2012 - 27 August, 2020 .

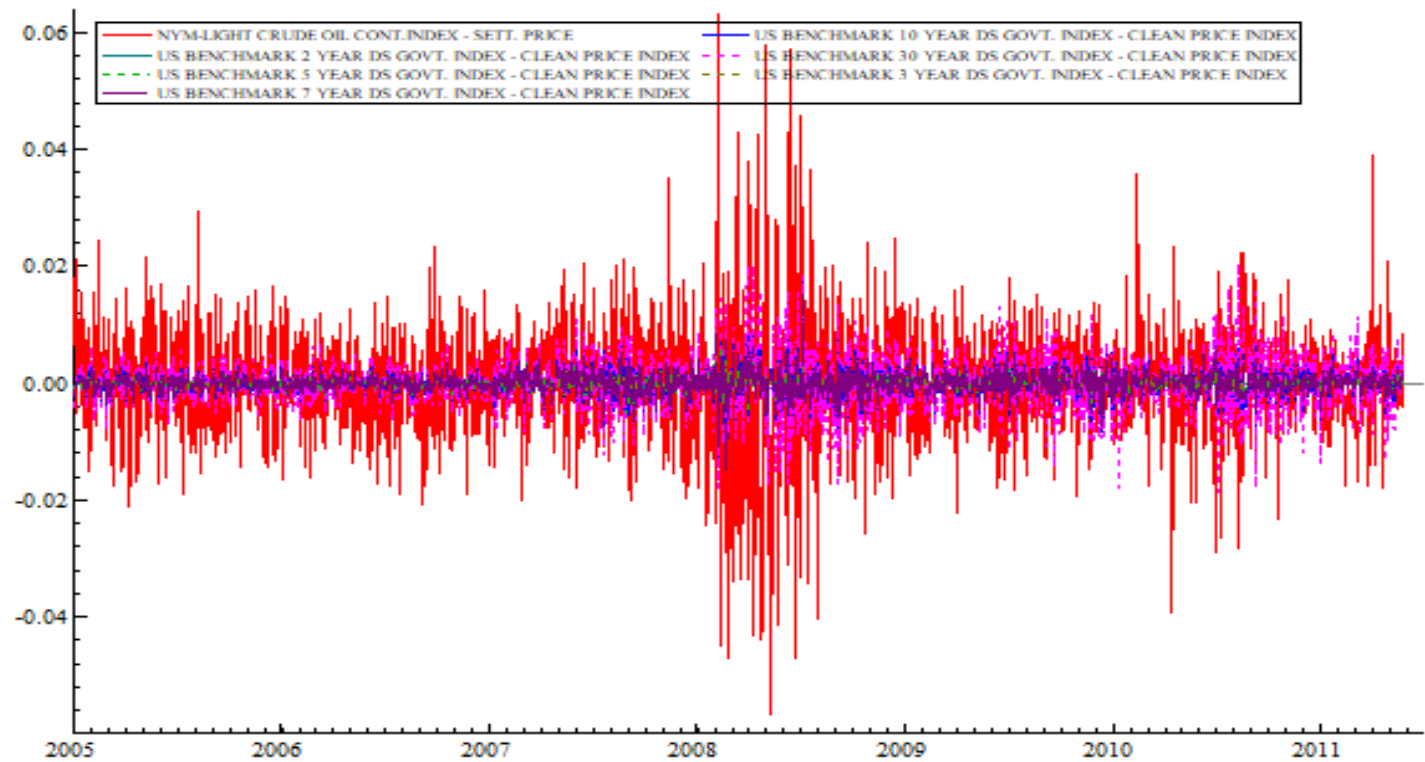

Fig-1: Actual series of the logarithmic returns of the markets, sample period: 3 January, 2005 - 31 August, 2012 


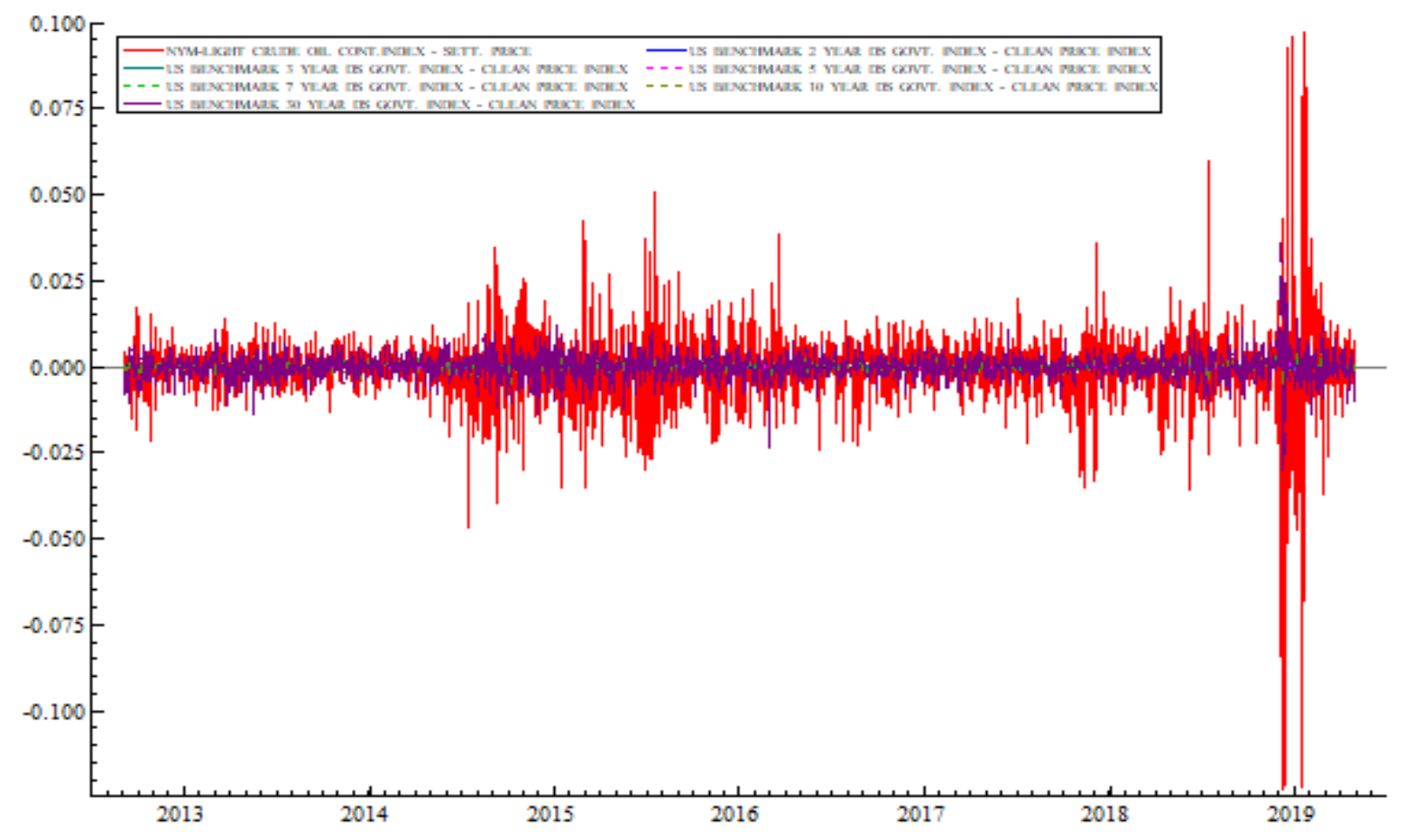

Fig-2: Actual series of the logarithmic returns of the markets, sample period: 3 September, 2012 - 27 August, 2020

Figure 3 and 4 plot the conditional variances for all market logarithmic returns in the two under investigation periods. Variances are extreme volatilite during both periods. Interestingly, we see a common movement.

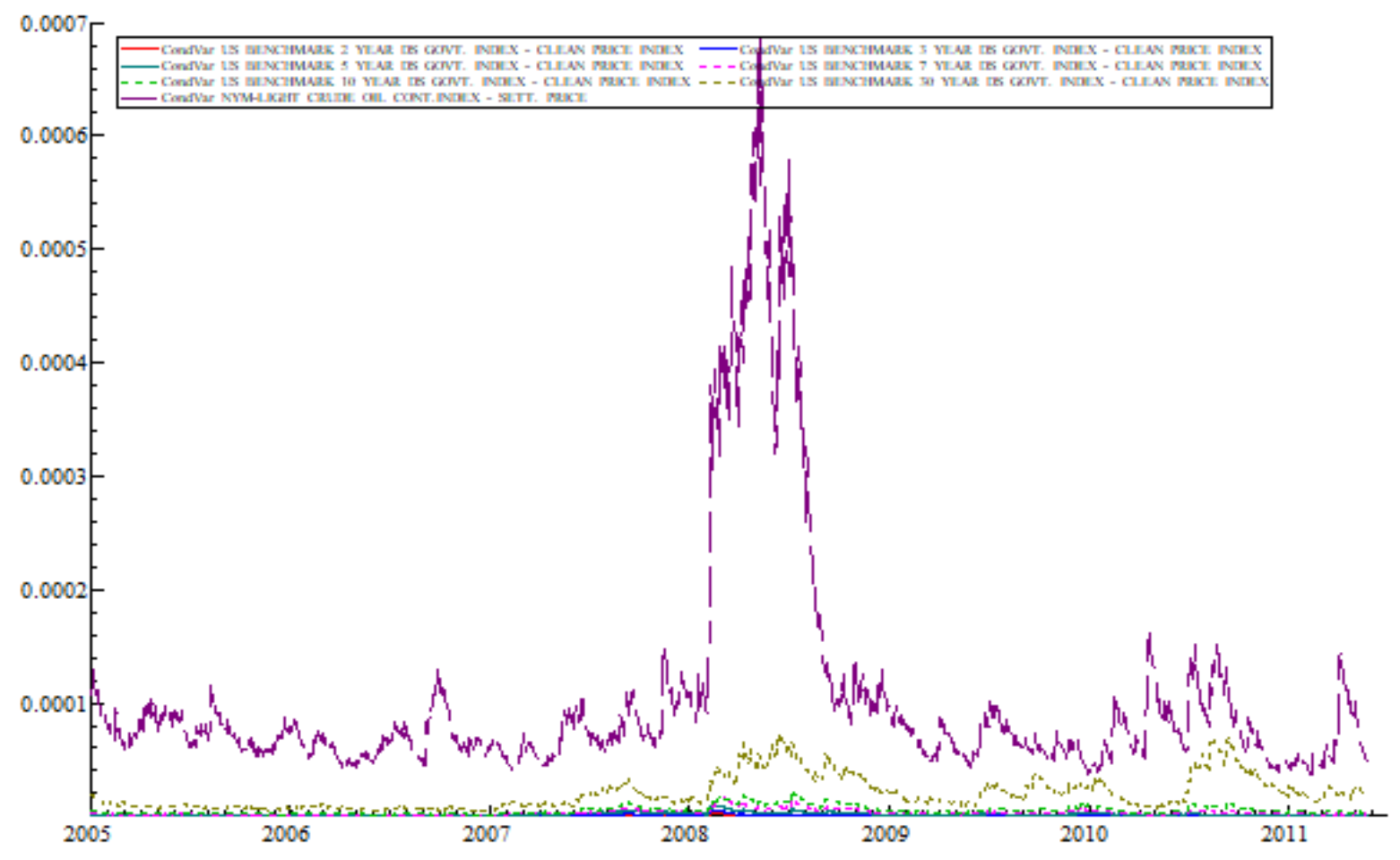

Fig-3: Conditional variances of the univariate GARCH (1,1) model, sample period: 3 January, 2005 - 31 August, 2012 


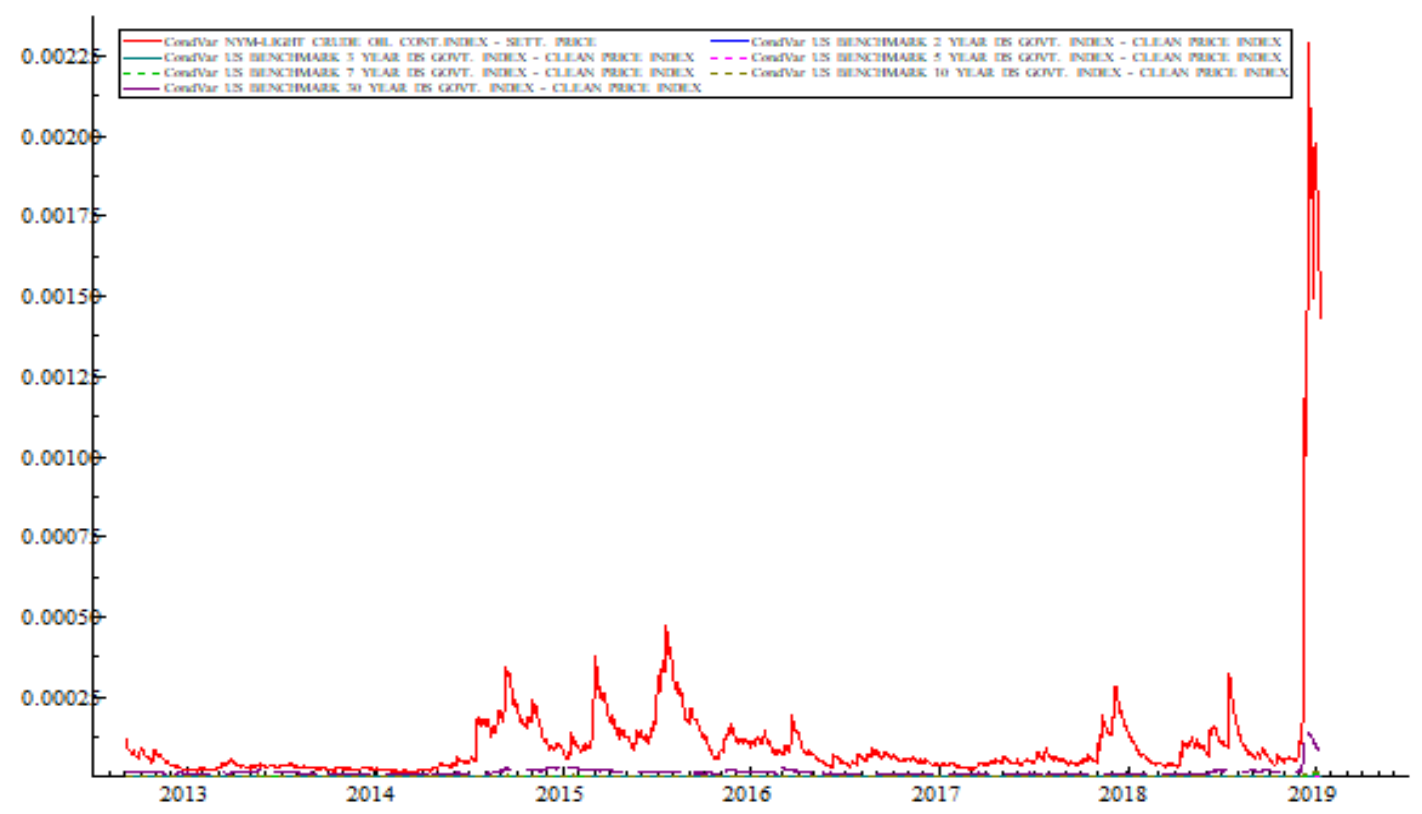

Fig-4: Conditional variances of the univariate GARCH (1,1) model, sample period: 3 September, 2012 - 27 August, 2020

\section{Sample period: 3 January, 2005 - 31 August, 2012}

In Tables 1 to 3 we report the estimates of the univariate $\operatorname{GARCH}(1,1)$ model We see significant $\mu$ value only for US BENCHMARK 7 YEAR DS GOVT.
INDEX - CLEAN PRICE INDEX. Constant $\omega$ is significant for all markets. In addition, all the ARCH (a) and GARCH (b) terms are highly significant for all markets.

Table-1: Estimates of univariate GARCH (1,1) model, sample period: 3 January, 2005 - 31 August, 2012

\begin{tabular}{|l|l|l|l|}
\hline & $\begin{array}{l}\text { NYM-LIGHT CRUDE OIL } \\
\text { CONT.INDEX - SETT. } \\
\text { PRICE }\end{array}$ & $\begin{array}{l}\text { US BENCHMARK 2 YEAR DS } \\
\text { GOVT. INDEX - CLEAN PRICE } \\
\text { INDEX }\end{array}$ & $\begin{array}{l}\text { US BENCHMARK 3 YEAR DS } \\
\text { GOVT. INDEX - CLEAN PRICE } \\
\text { INDEX }\end{array}$ \\
\hline constant $(\boldsymbol{\mu})$ & $0,000316^{*}$ & 0,0000028 & 0,0000081 \\
\hline t-Statistic & 1,693 & 0,2718 & 0,6418 \\
\hline p-Value & 0,0906 & 0,7858 & 0,5211 \\
\hline constant $(\boldsymbol{\omega})$ & $0,012796^{* *}$ & $0,000169^{*}$ & $0,001208^{*}$ \\
\hline t-Statistic & 2,564 & 1,653 & 1,364 \\
\hline p-Value & 0,0104 & 0,0984 & 0,1728 \\
\hline ARCH $(\mathbf{a})$ & $0,051460^{* * *}$ & $0,062458^{* * *}$ & $0,055245^{* * *}$ \\
\hline t-Statistic & 4,402 & 4,526 & 3,813 \\
\hline p-Value & 0,0000 & 0,0000 & 0,0001 \\
\hline GARCH $(\mathbf{b})$ & $0,935545^{* * *}$ & $0,938012^{* * *}$ & $0,942911^{* * *}$ \\
\hline t-Statistic & 64,28 & 76,87 & 65,77 \\
\hline p-Value & 0,0000 & 0,0000 & 0,0000 \\
\hline
\end{tabular}

Table-2: Estimates of univariate GARCH (1,1) model, sample period: 3 January, 2005 - 31 August, 2012

\begin{tabular}{|l|l|l|}
\hline & $\begin{array}{l}\text { US BENCHMARK 5 YEAR DS GOVT. } \\
\text { INDEX - CLEAN PRICE INDEX }\end{array}$ & $\begin{array}{l}\text { US BENCHMARK 7 YEAR DS GOVT. INDEX - } \\
\text { CLEAN PRICE INDEX }\end{array}$ \\
\hline constant $(\boldsymbol{\mu})$ & 0,0000172 & $0,0000353^{*}$ \\
\hline t-Statistic & 0,7413 & 1,099 \\
\hline p-Value & 0,4586 & 0,2720 \\
\hline constant $(\boldsymbol{\omega})$ & $0,008120^{*}$ & $0,015867 *$ \\
\hline t-Statistic & 1,604 & 1,838 \\
\hline p-Value & 0,1088 & 0,0661 \\
\hline ARCH $(\mathbf{a})$ & $0,042338^{*} * *$ & $0,038444^{*} * *$ \\
\hline t-Statistic & 3,070 & 3,535 \\
\hline p-Value & 0,0022 & 0,0004 \\
\hline GARCH $(\mathbf{b})$ & $0,952285^{*} * *$ & $0,956322^{*} * *$ \\
\hline t-Statistic & 60,62 & 78,39 \\
\hline p-Value & 0,0000 & 0,0000 \\
\hline
\end{tabular}


Table-3: Estimates of univariate GARCH (1,1) model, sample period: 3 January, 2005 - 31 August, 2012

\begin{tabular}{|l|l|l|}
\hline & $\begin{array}{l}\text { US BENCHMARK 10 YEAR DS GOVT. } \\
\text { INDEX - CLEAN PRICE INDEX }\end{array}$ & $\begin{array}{l}\text { US BENCHMARK 30 YEAR DS GOVT. } \\
\text { INDEX - CLEAN PRICE INDEX }\end{array}$ \\
\hline constant $(\boldsymbol{\mu})$ & 0,0000243 & 0,0000247 \\
\hline t-Statistic & 0,5926 & 0,3238 \\
\hline p-Value & 0,5535 & 0,7462 \\
\hline constant $(\boldsymbol{\omega})$ & $0,021935^{*}$ & $0,070534^{* *}$ \\
\hline t-Statistic & 1,907 & 2,029 \\
\hline p-Value & 0,0567 & 0,0426 \\
\hline ARCH $(\mathbf{a})$ & $0,041466^{* * *}$ & $0,045121^{* * *}$ \\
\hline t-Statistic & 4,894 & 6,353 \\
\hline p-Value & 0,0000 & 0,0000 \\
\hline GARCH $(\mathbf{b})$ & $0,954529^{* * *}$ & $0,951933^{* * * *}$ \\
\hline t-Statistic & 102,5 & 127,7 \\
\hline p-Value & 0,0000 & 0,0000 \\
\hline
\end{tabular}

Table-4: Estimates of the bivariate DCC-GARCH $(1,1)$ model, degrees of freedom, log-likelihood, sample period: 3 January, 2005 - 31 August, 2012

\begin{tabular}{|c|c|c|c|}
\hline & $\begin{array}{l}\text { NYM-LIGHT CRUDE OIL } \\
\text { CONT.INDEX - SETT. } \\
\text { PRICE-US BENCHMARK } 2 \\
\text { YEAR DS GOVT. INDEX - } \\
\text { CLEAN PRICE INDEX } \\
\end{array}$ & $\begin{array}{l}\text { NYM-LIGHT CRUDE OIL } \\
\text { CONT.INDEX - SETT. } \\
\text { PRICE- US BENCHMARK } 3 \\
\text { YEAR DS GOVT. INDEX - } \\
\text { CLEAN PRICE INDEX } \\
\end{array}$ & $\begin{array}{l}\text { NYM-LIGHT CRUDE OIL } \\
\text { CONT.INDEX - } \\
\text { PRICE- US BENCHMARK } \\
\text { YEAR DS GOVT. INDEX - } \\
\text { CLEAN PRICE INDEX } \\
\end{array}$ \\
\hline rho & 0,060952 & 0,046963 & $-0,200572 *$ \\
\hline t-Statistic & 0,1503 & 0,2371 & $-1,731$ \\
\hline p-Value & 0,8805 & 0,8126 & 0,0836 \\
\hline alpha $(\alpha)$ & 0,007692 & $0,008767 *$ & $0,016884 * *$ \\
\hline t-Statistic & 0,8772 & 1,554 & 2,731 \\
\hline p-Value & 0,3805 & 0,1204 & 0,0064 \\
\hline beta $(\beta)$ & $0,992298 * * *$ & $0,991223 * * *$ & $0,979673 * * *$ \\
\hline t-Statistic & 81,24 & 143,4 & 102,1 \\
\hline p-Value & 0,0000 & 0,0000 & 0,0000 \\
\hline degrees of freedom $(\mathrm{df})$ & $7,995271 * * *$ & $8,317341 * * *$ & $9,844983 * * *$ \\
\hline t-Statistic & 7,365 & 7,619 & 6,618 \\
\hline p-Value & 0,0000 & 0,0000 & 0,0000 \\
\hline log-likelihood & 19794,136 & 18659,095 & 17372,988 \\
\hline
\end{tabular}

Table-5: Estimates of the bivariate DCC-GARCH $(1,1)$ model, degrees of freedom, log-likelihood, sample period: 3 January, 2005 - 31 August, 2012

\begin{tabular}{|c|c|c|c|}
\hline & $\begin{array}{l}\text { NYM-LIGHT CRUDE OIL } \\
\text { CONT.INDEX - SETT. } \\
\text { PRICE-US BENCHMARK } \\
7 \text { YEAR DS GOVT. INDEX } \\
\text { - CLEAN PRICE INDEX }\end{array}$ & $\begin{array}{l}\text { NYM-LIGHT CRUDE OIL } \\
\text { CONT.INDEX - SETT. } \\
\text { PRICE- US BENCHMARK } \\
10 \text { YEAR DS GOVT. INDEX } \\
\text { - CLEAN PRICE INDEX }\end{array}$ & $\begin{array}{l}\text { NYM-LIGHT CRUDE OIL } \\
\text { CONT.INDEX - SETT. } \\
\text { PRICE- US BENCHMARK } \\
\text { 30 YEAR DS GOVT. INDEX } \\
\text { - CLEAN PRICE INDEX }\end{array}$ \\
\hline rho & $-0,221896^{*}$ & $-0,230786^{* *}$ & $-0,241048 * *$ \\
\hline t-Statistic & $-1,956$ & $-2,338$ & $-2,484$ \\
\hline p-Value & 0,0506 & 0,0195 & 0,0131 \\
\hline alpha $(\alpha)$ & $0,017240 * *$ & $0,020046 * * *$ & $0,018622 * * *$ \\
\hline t-Statistic & 2,680 & 3,060 & 3,215 \\
\hline p-Value & 0,0074 & 0,0022 & 0,0013 \\
\hline beta $(\beta)$ & $0,979344 * * *$ & $0,975134 * * *$ & $0,976781 * * *$ \\
\hline t-Statistic & 102,0 & 97,86 & 107,3 \\
\hline p-Value & 0,0000 & 0,0000 & 0,0000 \\
\hline $\begin{array}{l}\text { degrees of } \\
\text { freedom }(\mathrm{df})\end{array}$ & $10,215849 * * *$ & $10,799979 * * *$ & $12,182784 * * *$ \\
\hline t-Statistic & 6,271 & 6,190 & 5,685 \\
\hline p-Value & 0,0000 & 0,0000 & 0,0000 \\
\hline log-likelihood & 16707,576 & 16250,902 & 14952,826 \\
\hline
\end{tabular}




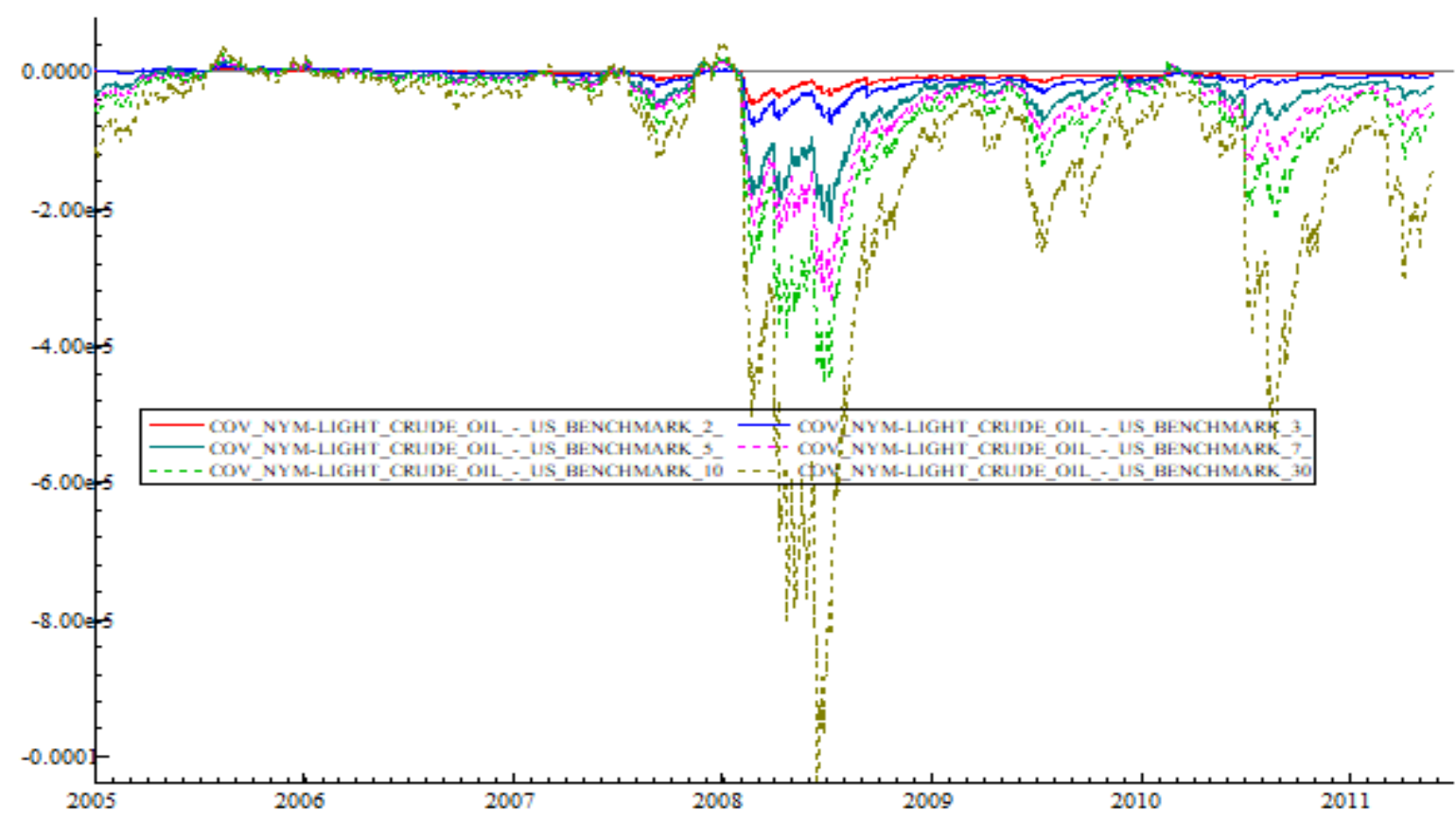

Fig-5: Conditional covariances of the univariate GARCH (1,1) model, sample period: 3 January, 2005 - 31 August, 2012

Tables 4 and 5 present the estimates of the bivariate DCC model. Average correlation is significant for all the pairs of markets except the cases of NYMLIGHT CRUDE OIL CONT.INDEX - SETT. PRICEUS BENCHMARK 2 YEAR DS GOVT. INDEX CLEAN PRICE INDEX and NYM-LIGHT CRUDE OIL CONT.INDEX - SETT. PRICE- US BENCHMARK 3 YEAR DS GOVT. INDEX - CLEAN PRICE INDEX. As shown in tables, $\alpha$ and $\beta$ are significant, showing strong ARCH and GARCH effects, except the insignificant ARCH effects of NYM-LIGHT CRUDE OIL CONT.INDEX - SETT. PRICE-US BENCHMARK 2 YEAR DS GOVT. INDEX - CLEAN PRICE INDEX. We also see the estimates of the degrees of freedom (v) and of the log-likelihood.
In Figure-5, we see the conditional covariances for all the pairs of market logarithmic returns. Covariances present a tremble trend and are extreme volatile in 2008 and after. In addition, they have mostly negative values.

Tables 6 and 7 shows the estimates of $x^{2}(12)$ statistic. Results do not accept the hypothesis of the absence of spillovers at $1 \%$ significance level. Hypothesis testing results and information criteria are exhibited in the two tables. Following Hosking [25] and $\mathrm{Li}$ and McLeod [26], the multivariate diagnostic tests detect no serial correlation on squared standardized residuals. Also, in Tables 6 and 7 we present the estimates of AIC and SIC information criteria.

Table-6: Diagnostic tests and information criteria, sample period: 3 January, 2005 - 31 August, 2012

\begin{tabular}{|c|c|c|c|}
\hline & 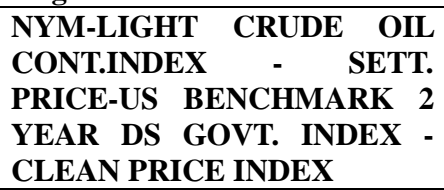 & $\begin{array}{l}\text { NYM-LIGHT CRUDE OIL } \\
\text { CONT.INDEX - SETT. PRICE- } \\
\text { US BENCHMARK } 3 \text { YEAR } \\
\text { DS GOVT. INDEX - CLEAN } \\
\text { PRICE INDEX }\end{array}$ & $\begin{array}{l}\text { NYM-LIGHT CRUDE OIL } \\
\text { CONT.INDEX - SETT. PRICE- } \\
\text { US BENCHMARK } 5 \text { YEAR } \\
\text { DS GOVT. INDEX - CLEAN } \\
\text { PRICE INDEX }\end{array}$ \\
\hline$x^{2}(4)$ & $1359,4 * *$ & $443,75 * *$ & $244,88 * *$ \\
\hline p-Value & 0,0000 & 0,0000 & 0,0000 \\
\hline Hosking $^{2}$ (50) & 149,666 & 191,808 & 201,207 \\
\hline p-Value & 0,9956837 & 0,6106019 & 0,4231769 \\
\hline Li-McLeod $^{2}(50)$ & 150,304 & 192,255 & 201,492 \\
\hline p-Value & 0,9951681 & 0,6017671 & 0,4176548 \\
\hline Akaike & 0,002103 & 0,002670 & 0,003314 \\
\hline Schwarz & 0,035708 & 0,036276 & 0,036919 \\
\hline
\end{tabular}


Table-7: Diagnostic tests and information criteria, sample period: 3 January, 2005 - 31 August, 2012

\begin{tabular}{|c|c|c|c|}
\hline & $\begin{array}{l}\text { NYM-LIGHT CRUDE OIL } \\
\text { CONT.INDEX - SETT. PRICE- } \\
\text { US BENCHMARK } 7 \text { YEAR DS } \\
\text { GOVT. INDEX - CLEAN } \\
\text { PRICE INDEX }\end{array}$ & $\begin{array}{l}\text { NYM-LIGHT CRUDE OIL } \\
\text { CONT.INDEX - SETT. PRICE- } \\
\text { US BENCHMARK 10 YEAR } \\
\text { DS GOVT. INDEX - CLEAN } \\
\text { PRICE INDEX }\end{array}$ & $\begin{array}{l}\text { NYM-LIGHT CRUDE OIL } \\
\text { CONT.INDEX - SETT. PRICE- } \\
\text { US BENCHMARK 30 YEAR } \\
\text { DS GOVT. INDEX - CLEAN } \\
\text { PRICE INDEX }\end{array}$ \\
\hline $\mathrm{x}^{2}(\mathbf{4})$ & $247,96 * *$ & $186,51 * *$ & $122,93 * *$ \\
\hline p-Value & 0,0000 & 0,0000 & 0,0000 \\
\hline Hosking $^{2}$ (50) & 201,193 & 184,428 & 211,385 \\
\hline p-Value & 0,4234518 & 0,7468346 & 0,2446697 \\
\hline Li-McLeod $^{2}(50)$ & 201,359 & 184,568 & 211,119 \\
\hline p-Value & 0,4202317 & 0,7444735 & 0,2487072 \\
\hline Akaike & 0,003646 & 0,003875 & 0,004524 \\
\hline Schwarz & 0,037252 & 0,037480 & 0,038129 \\
\hline
\end{tabular}

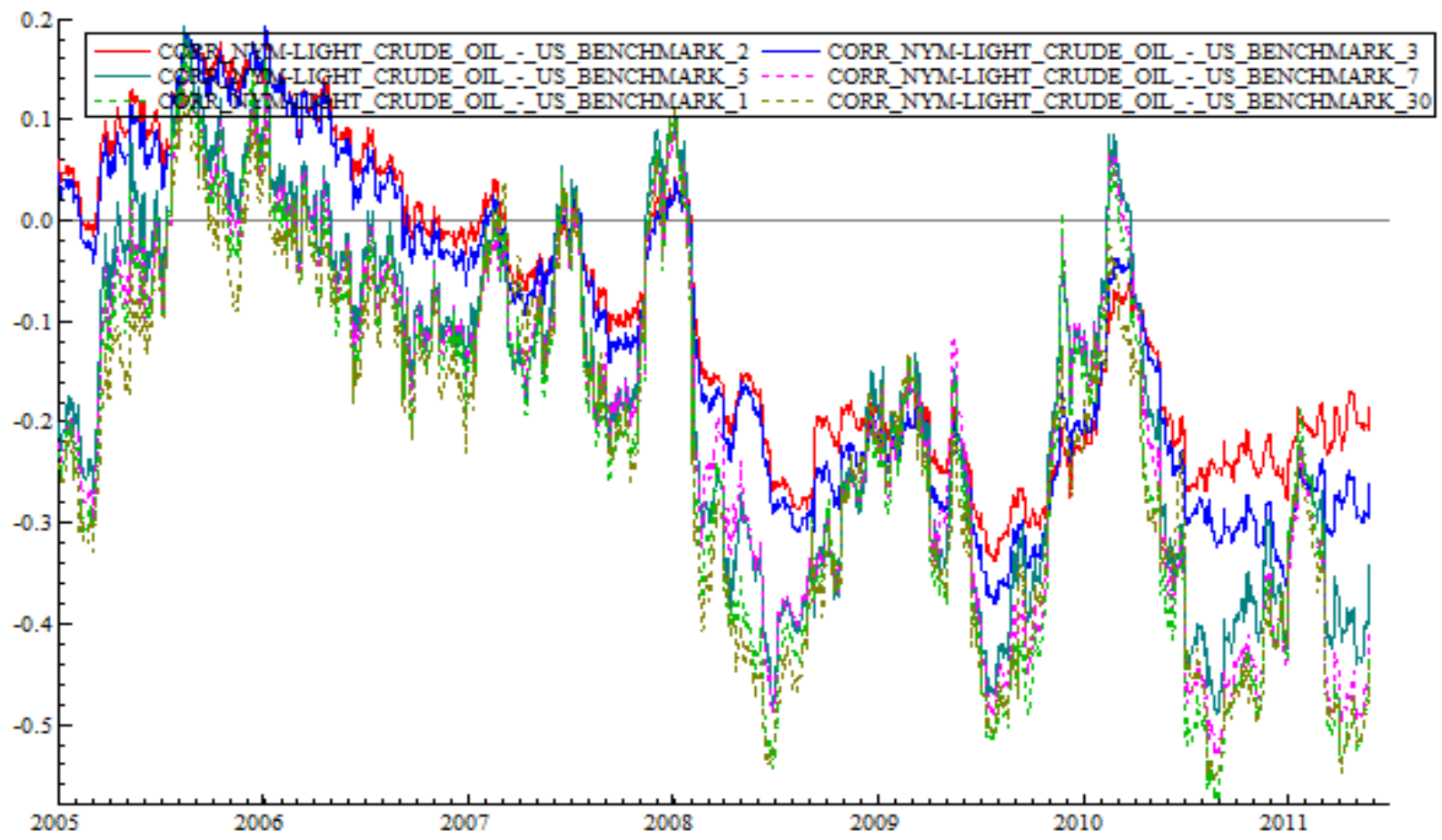

Fig-6: Dynamic conditional correlations of the bivariate DCC-GARCH (1,1) model, sample period: 3 January, 2005 - 31 August, 2012

Figure 6 shows the dynamic conditional correlations (DCCs) between time series. DCCs are extremely volatile. Interestingly, DCCs were seen to be persistently high and positive with some jumps over time. Positive and high correlation implies a less reliable stability of the correlation and that the benefit from market-portfolio diversification diminishes. In what follows, we can recognize the effects of major economic crises on the graph by taking into consideration the numerous picks and troughs: i.e. (1) the Lehman Brother bankruptcy on 15/9/2008, (2) the USA presidential election on $4 / 11 / 2008$, and (3)
Standard \& Poor's credit rating agency downgraded the credit rating of the USA from AAA to AA+ on $5 / 8 / 2011$.

Sample period: 3 September, 2012 - 27 August, 2020

Tables 8 to 10 present the estimates of the univariate $\operatorname{GARCH}(1,1)$ model. $\mu$ is significant only for US BENCHMARK 3 YEAR DS GOVT. INDEX CLEAN PRICE INDEX and US BENCHMARK 30 YEAR DS GOVT. INDEX - CLEAN PRICE INDEX. Constant $\omega$ is significant for all markets. ARCH (a) and GARCH (b) terms are significant for all markets. 
Table-8: Estimates of univariate GARCH $(1,1)$ model, sample period: 3 September, 2012 - 27 August, 2020

\begin{tabular}{|l|l|l|l|}
\hline & $\begin{array}{l}\text { NYM-LIGHT CRUDE } \\
\text { OIL CONT.INDEX - } \\
\text { SETT. PRICE }\end{array}$ & $\begin{array}{l}\text { US BENCHMARK 2 YEAR } \\
\text { DS GOVT. INDEX - CLEAN } \\
\text { PRICE INDEX }\end{array}$ & $\begin{array}{l}\text { US BENCHMARK 3 YEAR } \\
\text { DS GOVT. INDEX - CLEAN } \\
\text { PRICE INDEX }\end{array}$ \\
\hline constant $(\boldsymbol{\mu})$ & 0,000074 & $-0,0000056$ & $-0,0000129^{*}$ \\
\hline t-Statistic & 0,5172 & $-0,6970$ & $-1,275$ \\
\hline p-Value & 0,6051 & 0,4859 & 0,2024 \\
\hline constant $(\boldsymbol{\omega})$ & $0,004325 * *$ & $0,0001087^{*}$ & $0,000887^{*}$ \\
\hline t-Statistic & 2,019 & 1,399 & 1,144 \\
\hline p-Value & 0,0436 & 0,1619 & 0,2528 \\
\hline ARCH $(\mathbf{a})$ & $0,065060^{* * *}$ & $0,066304 * * *$ & $0,048292^{* * *}$ \\
\hline t-Statistic & 5,987 & 5,403 & 4,016 \\
\hline p-Value & 0,0000 & 0,0000 & 0,0001 \\
\hline GARCH $(\mathbf{b})$ & $0,937153^{* * *}$ & $0,939544 * * *$ & $0,951247 * * *$ \\
\hline t-Statistic & 95,66 & 88,64 & 68,66 \\
\hline p-Value & 0,0000 & 0,0000 & 0,0000 \\
\hline
\end{tabular}

Table-9: Estimates of univariate GARCH (1,1) model, sample period: 3 September, 2012 - 27 August, 2020

\begin{tabular}{|l|l|l|}
\hline & $\begin{array}{l}\text { US BENCHMARK 5 YEAR DS GOVT. } \\
\text { INDEX - CLEAN PRICE INDEX }\end{array}$ & $\begin{array}{l}\text { US BENCHMARK 7 YEAR DS GOVT. } \\
\text { INDEX - CLEAN PRICE INDEX }\end{array}$ \\
\hline constant $(\boldsymbol{\mu})$ & $-0,0000161$ & $-0,0000002$ \\
\hline t-Statistic & 0,9056 & $-0,01089$ \\
\hline p-Value & 0,3653 & 0,9913 \\
\hline constant $(\boldsymbol{\omega})$ & $0,007142^{*}$ & $0,017218^{*}$ \\
\hline t-Statistic & 1,322 & 1,399 \\
\hline p-Value & 0,1862 & 0,1620 \\
\hline ARCH $(\mathbf{a})$ & $0,038931^{* * *}$ & $0,034960^{* * *}$ \\
\hline t-Statistic & 3,472 & 3,491 \\
\hline p-Value & 0,0005 & 0,0005 \\
\hline GARCH $(\mathbf{b})$ & $0,954568^{* * *}$ & $0,956789^{* * *}$ \\
\hline t-Statistic & 58,57 & 60,78 \\
\hline p-Value & 0,0000 & 0,0000 \\
\hline
\end{tabular}

Table-10: Estimates of univariate GARCH $(1,1)$ model, sample period: 3 September, 2012 - 27 August, 2020

\begin{tabular}{|l|l|l|}
\hline & $\begin{array}{l}\text { US BENCHMARK 10 YEAR DS GOVT. } \\
\text { INDEX - CLEAN PRICE INDEX }\end{array}$ & $\begin{array}{l}\text { US BENCHMARK 30 YEAR DS GOVT. } \\
\text { INDEX - CLEAN PRICE INDEX }\end{array}$ \\
\hline constant $(\boldsymbol{\mu})$ & 0,0000133 & $0,0000917^{*}$ \\
\hline t-Statistic & 0,3759 & 1,252 \\
\hline p-Value & 0,7070 & 0,2106 \\
\hline constant $(\boldsymbol{\omega})$ & $0,027560^{*}$ & $0,107850^{*}$ \\
\hline t-Statistic & 1,563 & 1,956 \\
\hline p-Value & 0,1181 & 0,0506 \\
\hline ARCH $(\mathbf{a})$ & $0,033339^{* * *}$ & $0,032141^{* * *}$ \\
\hline t-Statistic & 4,067 & 4,936 \\
\hline p-Value & 0,0000 & 0,0000 \\
\hline GARCH $(\mathbf{b})$ & $0,959401^{* * *}$ & $0,962244^{* * *}$ \\
\hline t-Statistic & 77,44 & 114,7 \\
\hline p-Value & 0,0000 & 0,0000 \\
\hline
\end{tabular}

Tables 11 and 12 show the estimates of the bivariate DCC process. Average correlation is significant for all the pairs of markets. As presented in tables, $\alpha$ and $\beta$ are significant, implying strong ARCH and GARCH effects. In addition, we observe the estimates of the degrees of freedom (v) and of the loglikelihood.
Figure-7 graphs the estimated conditional covariances. They are extreme volatile and have mostly negative values. Interestingly, the pair of markets NYM-LIGHT_CRUDE_OIL-US_BENSCHMARK_10 present the most volatile covariance among the covariances for all the pairs of markets. 
Table-11: Estimates of the bivariate DCC-GARCH $(1,1)$ model, degrees of freedom, log-likelihood, sample period: 3 September, 2012 - 27 August, 2020

\begin{tabular}{|c|c|c|c|}
\hline & 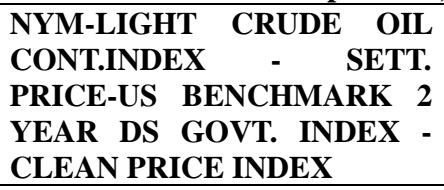 & 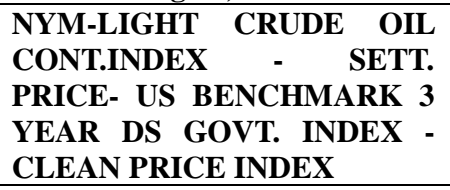 & $\begin{array}{l}\text { NYM-LIGHT } \\
\text { CRUDE } \\
\text { CONT.INDEX } \\
\text { PRICE- US BENCHMARK } 5 \\
\text { YEAR DS GOVT. INDEX } \\
\text { CLEAN PRICE INDEX } \\
\end{array}$ \\
\hline rho & $-0,156709 * * *$ & $-0,157951 * * *$ & $-0,179411 * * *$ \\
\hline t-Statistic & $-3,504$ & $-3,573$ & $-4,476$ \\
\hline p-Value & 0,0005 & 0,0004 & 0,0000 \\
\hline alpha $(\alpha)$ & $0,003644 *$ & $0,004562 * *$ & $0,004683^{*}$ \\
\hline t-Statistic & 1,940 & 2,158 & 1,886 \\
\hline p-Value & 0,0525 & 0,0311 & 0,0594 \\
\hline $\operatorname{beta}(\beta)$ & $0,993091 * * *$ & $0,991191 * * *$ & $0,989257 * * *$ \\
\hline t-Statistic & 293,8 & 330,4 & 254,6 \\
\hline p-Value & 0,0000 & 0,0000 & 0,0000 \\
\hline $\begin{array}{l}\text { degrees of freedom } \\
\text { (df) }\end{array}$ & $5,076738 * * *$ & $5,785807 * * *$ & $6,117417 * * *$ \\
\hline t-Statistic & 11,14 & 10,41 & 10,06 \\
\hline p-Value & 0,0000 & 0,0000 & 0,0000 \\
\hline log-likelihood & 20893,822 & 19546,299 & 18128,215 \\
\hline
\end{tabular}

Table-12: Estimates of the bivariate DCC-GARCH $(1,1)$ model, degrees of freedom, log-likelihood, sample period: 3

September, 2012 - 27 August, 2020

\begin{tabular}{|c|c|c|c|}
\hline & $\begin{array}{l}\text { NYM-LIGHT CRUDE OIL } \\
\text { CONT.INDEX } \\
\text { PRICE-US BENCHMARK } 7 \\
\text { YEAR DS GOVT. INDEX - } \\
\text { CLEAN PRICE INDEX }\end{array}$ & 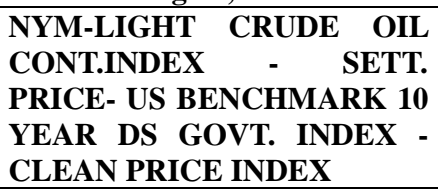 & $\begin{array}{l}\text { NYM-LIGHT CRUDE OIL } \\
\text { CONT.INDEX } \\
\text { PRICE- US BENCHMARK } 30 \\
\text { YEAR DS GOVT. INDEX - } \\
\text { CLEAN PRICE INDEX }\end{array}$ \\
\hline rho & $-0,190665 * * *$ & $-0,203642 * * *$ & $-0,208213 * * *$ \\
\hline t-Statistic & $-4,874$ & $-5,088$ & $-5,595$ \\
\hline p-Value & 0,0000 & 0,0000 & 0,0000 \\
\hline alpha $(\alpha)$ & $0,005010^{*}$ & $0,005607 *$ & $0,004901 *$ \\
\hline t-Statistic & 1,883 & 1,883 & 1,770 \\
\hline p-Value & 0,0598 & 0,0598 & 0,0768 \\
\hline beta $(\beta)$ & $0,988175 * * *$ & $0,986919 * * *$ & $0,987380 * * *$ \\
\hline t-Statistic & 232,1 & 175,8 & 193,6 \\
\hline p-Value & 0,0000 & 0,0000 & 0,0000 \\
\hline $\begin{array}{l}\text { degrees of freedom } \\
\text { (df) }\end{array}$ & $6,357867 * * *$ & $6,493858 * * *$ & $6,620931 * * *$ \\
\hline t-Statistic & 9,609 & 9,345 & 8,776 \\
\hline p-Value & 0,0000 & 0,0000 & 0,0000 \\
\hline log-likelihood & 17372,765 & 16810,690 & 15244,207 \\
\hline
\end{tabular}

Source: Datastream ${ }^{\circledR}$ Database 


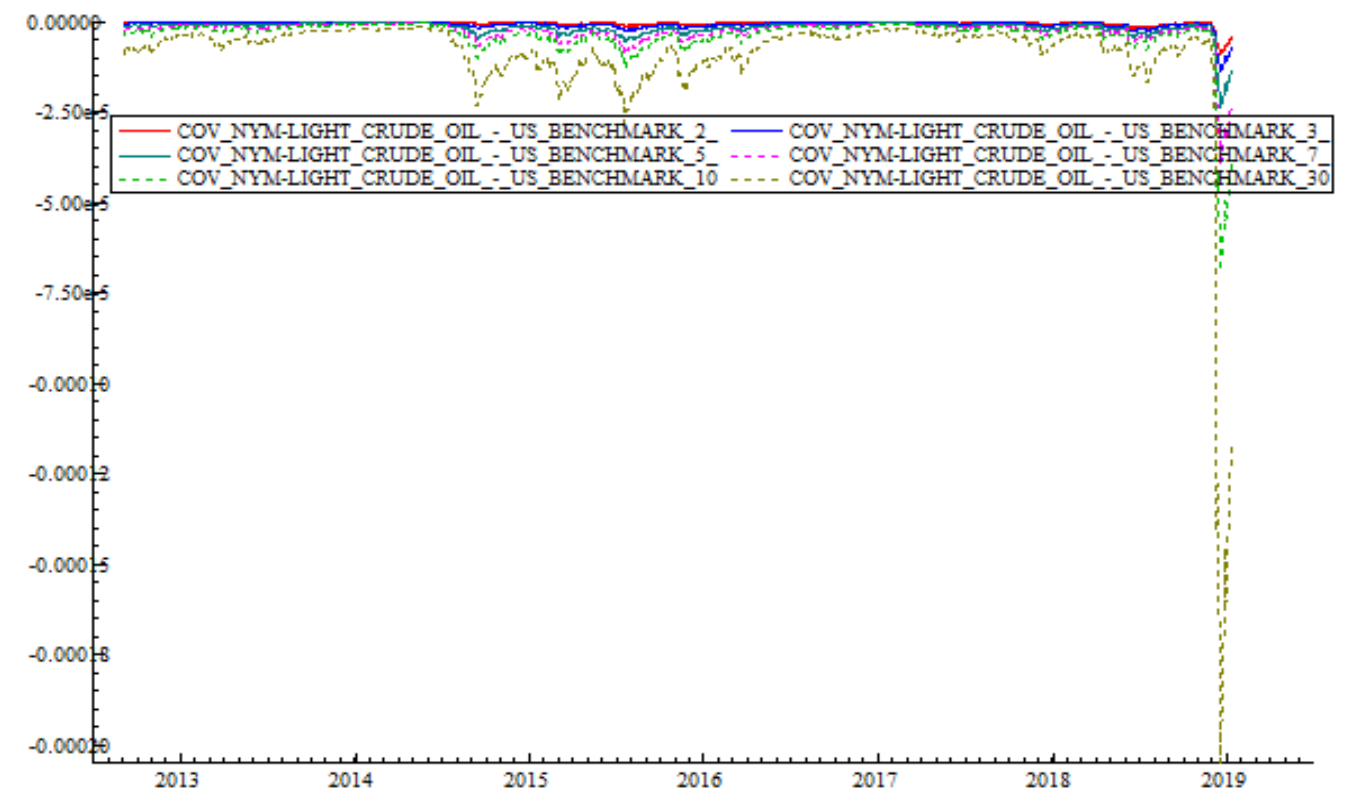

Fig-7: Conditional covariances of the univariate GARCH (1,1) model, sample period: 3 September, 2012 - 27 August, 2020

Tables 13 and 14 show that the estimated $\mathrm{x}^{2}$ (12) statistic imply the rejection of the null hypothesis of no spillover effects at $1 \%$. Estimates of
Hosking [25] and Li and McLeod [26] tests show no serial correlation the estimates of AIC and SIC information criteria are stated.

Table-13: Diagnostic tests and information criteria, sample period: 3 September, 2012 - 27 August, 2020

\begin{tabular}{|c|c|c|c|}
\hline & $\begin{array}{l}\text { NYM-LIGHT CRUDE OIL } \\
\text { CONT.INDEX - SETT. } \\
\text { PRICE-US BENCHMARK } 2 \\
\text { YEAR DS GOVT. INDEX - } \\
\text { CLEAN PRICE INDEX }\end{array}$ & $\begin{array}{l}\text { NYM-LIGHT CRUDE OIL } \\
\text { CONT.INDEX - SETT. } \\
\text { PRICE- US BENCHMARK } \\
3 \text { YEAR DS GOVT. INDEX - } \\
\text { CLEAN PRICE INDEX }\end{array}$ & $\begin{array}{l}\text { NYM-LIGHT CRUDE OIL } \\
\text { CONT.INDEX }- \text { SETT. } \\
\text { PRICE- US BENCHMARK } 5 \\
\text { YEAR DS GOVT. INDEX - } \\
\text { CLEAN PRICE INDEX }\end{array}$ \\
\hline$x^{2}(4)$ & $861,08 * *$ & $696,40 * *$ & $623,94 * *$ \\
\hline p-Value & 0,0000 & 0,0000 & 0,0000 \\
\hline Hosking $^{2}(50)$ & 232,246 & 228,028 & 254,407 \\
\hline p-Value & 0,0586849 & 0,0705267 & 0,0041997 \\
\hline Li-McLeod $^{2}(50)$ & 201,856 & 227,966 & 254,087 \\
\hline p-Value & 0,0607411 & 0,0709111 & 0,0043714 \\
\hline Akaike & 0,001504 & 0,002185 & 0,002902 \\
\hline Schwarz & 0,035262 & 0,035943 & 0,036660 \\
\hline
\end{tabular}

Table-14: Diagnostic tests and information criteria, sample period: 3 September, 2012 - 27 August, 2020

\begin{tabular}{|l|l|l|l|}
\hline & $\begin{array}{l}\text { NYM-LIGHT CRUDE OIL } \\
\text { CONT.INDEX - SETT. PRICE- } \\
\text { US BENCHMARK 7 YEAR } \\
\text { DS GOVT. INDEX - CLEAN } \\
\text { PRICE INDEX }\end{array}$ & $\begin{array}{l}\text { CONT.INDEX - SETT. PRICE- } \\
\text { US BENCHMARK 10 YEAR } \\
\text { DSOVT. INDEX - CLEAN } \\
\text { PRICE INDEX }\end{array}$ & $\begin{array}{l}\text { NYM-LIGHT CRUDE OIL } \\
\text { CONT.INDEX - SETT. PRICE- } \\
\text { DS GENCHMARK 30 YEAR } \\
\text { PRICE INDEX }\end{array}$ \\
\hline $\mathbf{x}^{\mathbf{2}(\mathbf{4})}$ & $604,07 * *$ & $615,43^{* *}$ & $904,36^{* *}$ \\
\hline p-Value & 0,0000 & 0,0000 & 0,0000 \\
\hline Hosking (50) & 219,226 & 220,535 & 227,388 \\
\hline p-Value & 0,1672193 & 0,1522965 & 0,0894029 \\
\hline $\begin{array}{l}\text { Li-McLeod } \\
(\mathbf{5 0})\end{array}$ & 218,998 & 220,246 & 227,012 \\
\hline p-Value & 0,1699185 & & 0,0922270 \\
\hline Akaike & 0,003284 & 0,1555091 & 0,004360 \\
\hline Schwarz & 0,037042 & 0,003568 & 0,038118 \\
\hline
\end{tabular}

In Figure-8, we see the DCCs between timevarying series. We observe that DCCs are extremely volatile and have mostly negative values with some time-varying jumps supporting that investors benefit from market-portfolio diversification. On the graph, we can clearly see the effects of major economic crises: i.e. (1) the European Central Bank announcement of an aggressive money-creation program, printing more than 
one trillion new euros on 22/01/2015, (2) the Black Monday on 24/08/2015, (3) the United Kingdom referendum on 23/06/2016, and (4) the Covid-19 pandemic on $03 / 2020$.

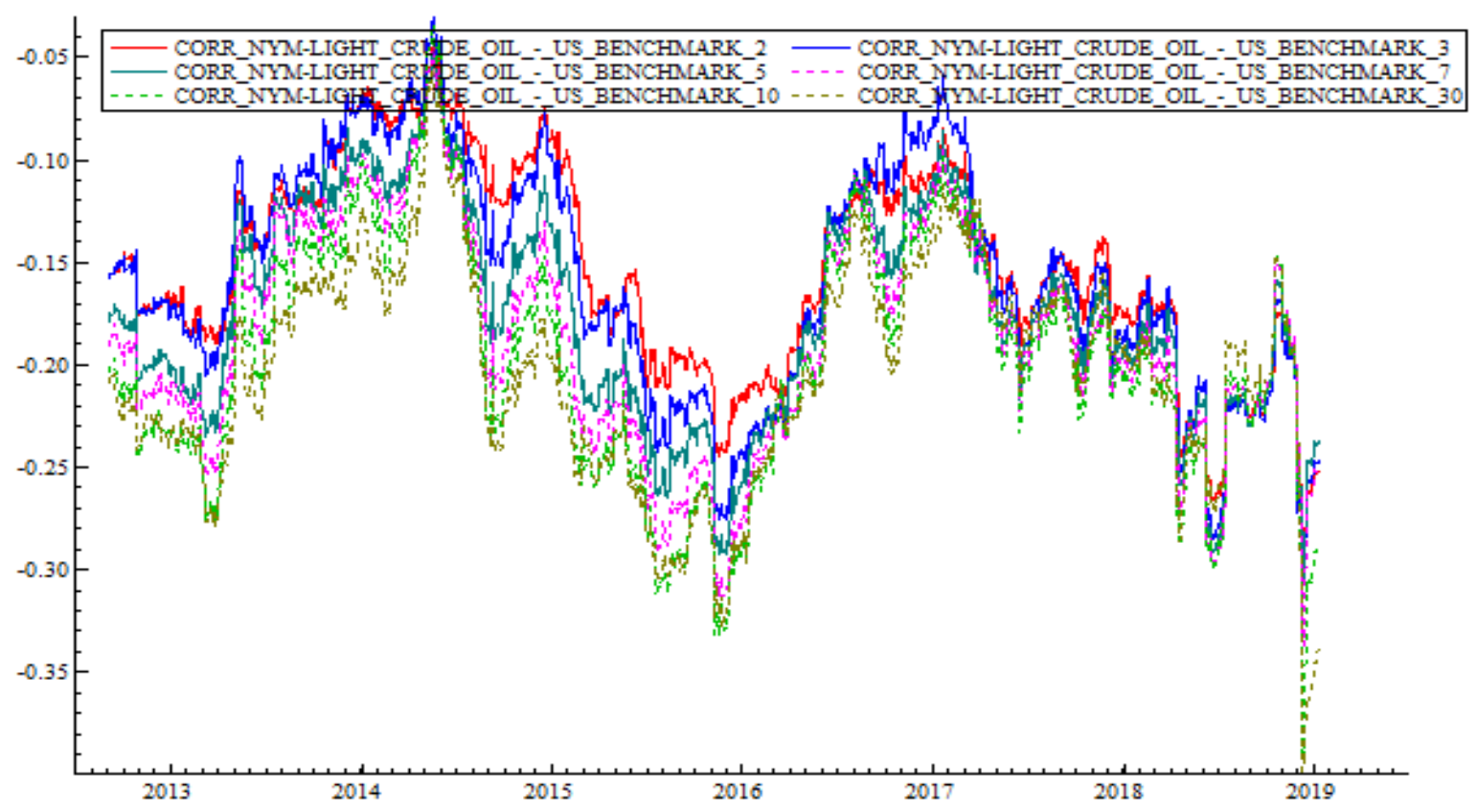

Fig-8: Dynamic conditional correlations of the bivariate DCC-GARCH (1,1) model, sample period: 3 September, 2012 - 27 August, 2020

\section{CONCLUSIONS}

This empirical paper is a contribution to the empirical literature on financial and derivative markets contagion effects. We examine the volatility transmission between Crude oil future and USA bond markets during the period 2005-2020. To measure volatility spillovers and contagion phenomenon, we use the multivariate DCC-GARCH process. We divide the under investigation period into sub-periods: 2005-2012 and 2012-2020. Empirical results suggest the existence of spillover effects during the both sub-periods. In the first sub-period, our results indicate the existence of contagion effect at the beginning of the period. Interestingly, during the second sub-period, there are no contagion effects.

A natural extension to this empirical research would be to investigate potential volatility spillover effects during the 2005-2020 period by taking into consideration different elements of financial analysis such as long memory and speed of market information.

\section{REFERENCES}

1. Engle, R F. (2002). Dynamic conditional correlation-a simple class of multivariate GARCH models. Journal of Business \& Economic Statistics, 20, 339-350.

2. Forbes, K. J., \& Rigobon, R. (2002). No Contagion, Only Interdependence: Measuring Stock Market Comovements. The Journal of Finance, 57(5), 2223-2261.

3. Hamilton, J. D. (2008). Oil and the macroeconomy, In: Durlauf, S., Blume, L. (Eds.),
The New Palgrave Dictionary of Economics, 2nd ed. Palgrave MacMillan Ltd.

4. Bernanke, B. S. (2006). "Energy and the economy," speech on June 15. Economic Club of Chicago.

5. Lee, K., \& Ni, S. (2002). On the dynamic effects of oil price shocks: a study using industry level data. J Monet Econ. 49(4), 823-852.

6. Hamilton, J. D. (2009). Causes and consequences of the oil shock of 2007-08. Brook. Pap Econ Act, 215-261 (Spring).

7. Kilian, L., \& Park, C. (2009). The impact of oil price shocks on the U.S. stock market. Int Econ Rev, 50, 1267-1287.

8. Filis, G., Degiannakis, S., \& Floros, C. (2011). Dynamic correlation between stock market and oil prices: The case of oil-importing and oil-exporting countries. Int Rev Financ Anal, 20, 152-164.

9. Basher, S. A., Haug, A. A., \& Sadorsky, P. (2012). Oil prices, exchange rates and emerging stock markets. Energy Econ, 34, 227-240.

10. Abhyankar, A., Xu, B., \& Wang, J. (2013). Oil price shocks and the stock market: evidence from Japan. Energy J, 34, 199-222. 
11. Degiannakis, S., Filis, G., \& Kizys, R. (2014). The effects of oil price shocks on stock market volatility: evidence from European data. Energy $J$, 35, 35-56.

12. Apergis, N., \& Miller, S. M. (2009). Do structural oil-market shocks affect stock prices? Energy Econ, 31, 569-575.

13. Wang, Y., Wua, C., \& Li, Y. (2013). Oil price shocks and stock market activities: Evidence from oil-importing and oil-exporting countries. J Comp Econ, 41(4), 1220-1239.

14. Park, J., \& Ratti, R. A. (2008). Oil prices and stock markets in the U.S. and 13 European countries. Energy Econ. 30, 2587-2608.

15. Bernanke, B. S., Gertler, M., \& Watson, M. (1997). Systematic monetary policy and the effects of oil price shocks. Brook. Pap Econ Act, 28, 91-157.

16. Kilian, L., \& Lewis, L. T. (2011). Does the Fed respond to oil price shocks? Econ J, 121, 10471072.

17. Haigh, M. S., \& Holt, M. T. (2002). Crack spread hedging: Accounting for time-varying volatility spillovers in the energy futures markets. Journal of Applied Econometrics, 17, 269-289. https://doi.org/10.1002/jae.628.

18. Guo, H., \& Kliesen, K. L. (2005). Oil price volatility and U.S. macroeconomic activity. Federal Reserve Bank of St. Louis Review, 87, 669-684. https://doi.org/10.20955/r.87.669-84.
19. Malik, F., \& Hammoudeh, S. (2007). Shock and volatility transmission in the oil, US and gulf equity markets. International Review of Economics and Finance, 16, 357-368. https://doi.org/10.1016/j.iref.2005.05.005.

20. Driesprong, G., Jacobsen, B., \& Maat, B. (2008). Striking oil: Another puzzle? Journal of Financial Economics, 89 , $307-327$. https://doi.org/10.1016/j.jfineco.2007.07.008

21. Geman, H., \& Kharoubi, C. (2008). WTI crude oil futures in portfolio diversification: The time-tomaturity effect. Journal of Banking and Finance, 32, 2553-2559.

22. Ewing, B. T., \& Malik, F. (2010). Estimating Volatility Persistence in Oil Prices Under Structural Breaks. The Financial Review, 45, 1011-1023

23. Wu, F., Guan, Z., \& Myers, R. J. (2011). Volatility spillover effects and cross hedging in corn and crude oil futures. Journal of Futures Markets, 31, 1052-1075.

24. Bollerslev, T. (1986). Generalized autoregressive conditional heteroskedasticity, Journal of Econometrics, 31(3), 307-327.

25. Hosking, J. R. M. (1980). The Multivariate Portmanteau Statistic. Journal of the American Statistical Association, 75(371), 602-608.

26. McLeod, A. I., \& Li, W. K. (1983). Diagnostic checking ARMA time series models using squared-residuals autocorrelations. Journal of Time Series Analysis, 4(4), 269-273. 